\title{
Thinning of antenna array via adaptive memetic particle swarm optimization
}

\author{
Xiu Zhang and Xin Zhang* (1)
}

\begin{abstract}
Massive multiple input multiple output antenna array is crucial for the fifth generation wireless communication. Proper antenna array design can reduce interference among different signals and generate desirable beamforming. Sparse antenna array is able to form narrower beam with lower sidelobe than equally spaced antenna array given the same number of array elements. However, determining the position of elements is non-deterministic polynomial-time hard. To effectively solve such problem, this paper proposes adaptive memetic particle swarm optimization (AMPSO) algorithm. The algorithm adaptively tunes algorithmic parameters of particle swarm optimization (PSO). Moreover, crossover operator is added to enhance local exploiting search information of PSO. Sparse antenna array design is modeled as a minimization by thinning method. It is then tackled by the proposed algorithm. In terms of peak sidelobe level, the AMPSO algorithm shows good performance compared with PSO and genetic algorithm.
\end{abstract}

Keywords: Sparse antenna array, Thinning array, Particle swarm optimization, Parameter control, Memetic computing

\section{Introduction}

Massive multiple input multiple output antenna array is crucial for the fifth generation wireless communication [1]. No matter in cognitive radio networks, ad hoc networks, or radar networks [2-5], antenna play an important role to ensure data transmission and high Quality of Services (QoS) under certain communication requirements [6-9]. The design of antenna arrays is well known to be a hard nonlinear programming problem [10]. Recently, many researchers attempt to create efficient and effective optimization algorithms to solve the design of antenna arrays. Stochastic algorithms are popularly used because traditional optimization methods are not suitable due to the unavailable of gradient information [11-13]. Stochastic algorithms also show good performance for such design problems $[14,15]$.

Stochastic algorithms consist of two classes: evolutionary algorithms (EAs) and swarm intelligence (SI) algorithms. Informally, EAs contain genetic algorithm (GA) [16], evolutionary strategies [17], and differential evolution [18]. These algorithms simulate the evolution of genetic process of livings. They usually contain

*Correspondence: ecemark@mail.tjnu.edu.cn

Tianjin Key Laboratory of Wireless Mobile Communications and Power

Transmission, Tianjin Normal University, Tianjin, China both mutation and crossover operators. SI algorithms contain particle swarm optimization (PSO) [19], artificial bee colony $(\mathrm{ABC})$ [20], neighborhood search optimization [21], etc. SI algorithms emulate the social behaviors of swarms or particles. They usually do not contain crossover operator. Although EAs and SI are created based on different nature, their positive combinations are able to result effective algorithms [22]. In the past, GA, PSO, and $\mathrm{ABC}$ have been used in antenna designs $[15,23,24]$. Recently, covariance matrix adaptation evolutionary strategy [25] and differential evolution [26] are applied to synthesize antenna array patterns.

A large number of iterations is needed for standard PSO algorithm to obtain a satisfactory solution [27]. This is not acceptable for users as antenna simulation often takes a long time. In this paper, standard PSO is modified by adding a crossover operator and a parameter adaptation method. The idea is reasonable as memetic with crossover operator can enlarge and promote algorithm's search; also parameter control is an effective way to make the algorithm adapt to different design problems and saves the fine-tuning efforts of users. The new algorithm is named as adaptive memetic particle swarm optimization (AMPSO), which is applied to tackle antenna design. Numerical
Springer Open

(c) The Author(s). 2017 Open Access This article is distributed under the terms of the Creative Commons Attribution 4.0 International License (http://creativecommons.org/licenses/by/4.0/), which permits unrestricted use, distribution, and reproduction in any medium, provided you give appropriate credit to the original author(s) and the source, provide a link to the Creative Commons license, and indicate if changes were made. 
experiment is conducted studying the effect of adding crossover and parameter adaptation. The results are discussed based on different metrics and compared with other algorithms.

In the following, Section 2 introduces the antenna array design considered in this paper. Section 3 gives standard PSO and the proposed AMPSO algorithm. Section 4 presents numerical simulation with discussions. Section 5 concludes the paper.

\section{Sparse antenna array and related works}

Sparse antenna array design can be classified to two categories. One refers to that array elements can be placed anywhere between the aperture of antenna array. The other is that thinning the distance to grids and positions in grids are equally spaced. In the first case, elements can be arbitrarily placed, hence the positions of elements are continuous variables. In the second case, elements could be placed on grids, hence its search space is discrete and finite. However, when the number of array elements increases up to tens or hundreds, search space of such combinatorial optimization problem exponentially increases, which brings huge trouble for conventional optimization algorithms.

Given a sparse linear antenna array, as shown in Fig. 1, the number of array elements is $N$ (labels above $z$ axis), the space between grids is $d$, and the number of grid positions along $z$ axis is $M$ (labels below $z$ axis). Hence, the aperture of this linear antenna array is $(M-1) d$. To keep the same aperture, two array elements have to be placed on the first and the last positions of grids. In this model, the number of variables is $N-2$ and there are candidate $M-2$ positions to be placed. Suppose all array elements have no order or priorities, they have the same incentive amplitude and phase distribution. Thus, the total number of combinations of elements is:

$$
C_{(M-2)}^{(N-2)}=\frac{(M-2) !}{(N-2) !(M-N) !} .
$$

The thinned linear antenna array can be modeled as a minimization problem, whose objective is to minimize peak sidelobe level (PSLL) and maximize main lobe peak level. In this way, interference among elements can be greatly avoided, and the data transmission can

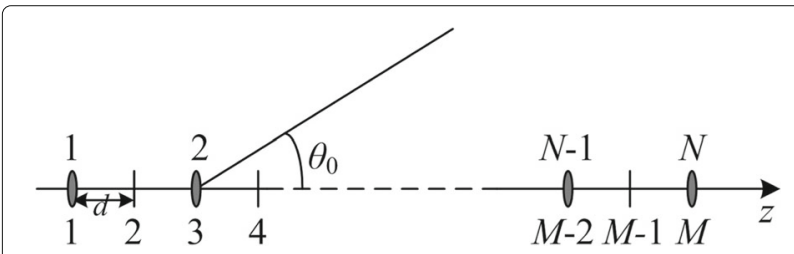

Fig. 1 Flow chart of sparse linear antenna array be realized in high array gain. The far field pattern is expressed as:

$$
E(\mathbf{u})=\sum_{i=1}^{M} \delta_{i} I_{i} e^{j \phi_{i}} e^{j k \mathbf{u} x_{i}} \operatorname{elpat}(i),
$$

where $\delta_{i}$ denotes whether the $i$ th grid is placed an element ( 1 for yes and 0 for no); $\mathbf{u}=\cos \theta-\cos \theta_{0}, I_{i}$ is the excitation amplitude of element $i$ and $\phi_{i}$ is the phase position of element $i$; $x_{i}$ denotes the element position, its distance from the first element is $(i-1) d ; \theta_{0}$ is the direction of main beam, and $\theta$ is the sweeping direction of linear antenna array; elpat $(i)$ is the direction pattern of element $i$. Suppose all elements have the same excitation amplitude and the same phase, then (6) is simplified to:

$$
E(\mathbf{u})=\sum_{i=1}^{M} \delta_{i} e^{j k \mathbf{u} x_{i}}
$$

Thus, the optimization model of this type array is:

$$
\begin{array}{ll}
\min f & =-\frac{|E(\mathbf{u})|}{|M P|} \\
\text { s.t. } & 1 \leq|\mathbf{u}| \leq \frac{\lambda C_{0}}{N d} \\
& \delta_{i} \in\{0,1\}, i=1,2, \ldots, M
\end{array}
$$

where $M P$ is mainlobe peak level, $C_{0}$ is a correction parameter as the first zero point may shift in nonuniform array. The constraint in (4) is to constrain the value of $\mathbf{u}$ so that mainlobe field is removed in computing $E(\mathbf{u})$.

Previously, many researches have reported in literature. Wang et al. considered nonuniform antenna array design for millimeter wave situations [28]. Zhao et al. studied resource allocation problem in MIMO systems such as time allocation [29] and power allocation [30].

\section{Optimization algorithms}

This section depicts standard PSO and the proposed AMPSO algorithm.

\subsection{Standard particle swarm optimization algorithm}

The main procedures of standard PSO is expressed in Fig. 2. It can be seen that the main loop of PSO is the updating of velocities and positions of particles. The number of particles is denoted as $N p$, which also means the size of population. As in initial population step, PSO starts by a set of randomly created particles in given search space. Usually, the evaluation of particles involves the interaction with problem. In this paper, the problem is nonuniform antenna design, which should be preset ahead of executing algorithm. Then, the main generation of standard PSO is executed. Compared with other swarm intelligence approaches, like $\mathrm{ABC}$, dragonfly algorithm and biogeography-based optimization, the procedures of PSO is more concise, though it contains more algorithmic parameters than others. 


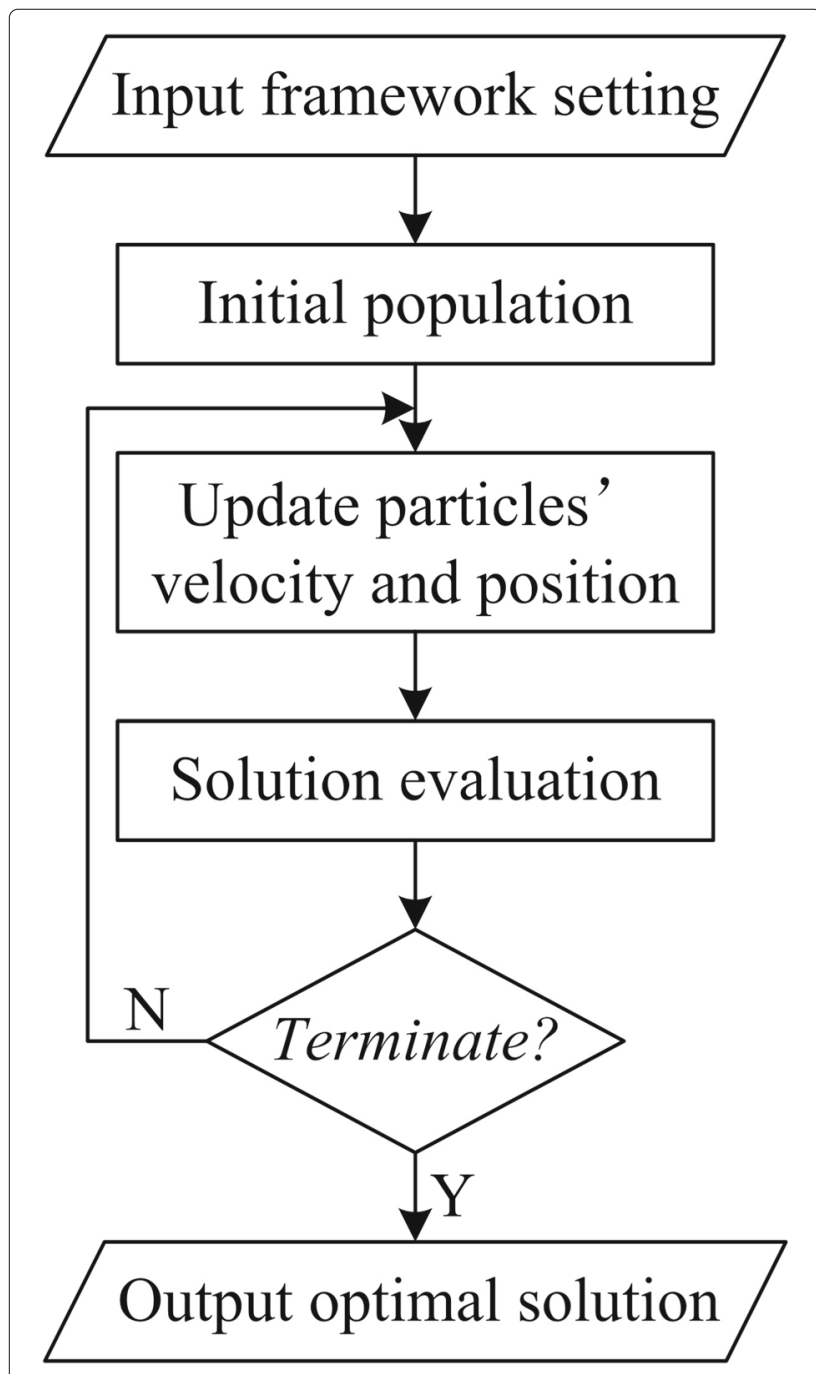

Fig. 2 Flow chart of standard particle swarm optimization algorithm

Let $D$ denote problem dimension, updating velocity and position of particle $i$ is realized by the following equation:

$$
v_{i j}(t+1)=w v_{i j}(t)+c_{1} r_{1}\left(p_{i j}-x_{i j}(t)\right)+c_{2} r_{1}\left(p_{g j}-x_{i j}(t)\right),
$$

$$
x_{i j}(t+1)=x_{i j}(t)+v_{i j}(t+1),
$$

where subscript $j \in[1, D]$ refers to the $j$ th dimension; $t$ refers to the $t$ th generation; $r_{1}$ and $r_{2}$ are random numbers between 0 and 1 . $\mathbf{p}_{i}$ and $\mathbf{p}_{g}$ are respectively personal best and global best positions that particle $i$ walked through. In this equation, $w, c_{1}$, and $c_{2}$ are three algorithmic parameters of PSO. Their setting for good performance of algorithm depends on the properties of practical problem. In other words, they are sensitive to problem types.

Recently, many researches have been published about parameter control of PSO. Inertia weight was adapted in a stability-based manner, which could improve the performance of PSO [31]. Khan et al. improved PSO by a random mutation mechanism and a dynamic inertia weight adaptive method, which could facilitate algorithm convergence [32]. Jin et al. improved PSO through enlarging explorative ability of PSO to design a permanent magnet synchronous machine [33].

\subsection{Adaptive memetic PSO algorithm}

As designated by no free lunch theory [34], the performance of an optimization algorithm could be improved by incorporating prior knowledge or properly hybrid with other search operators or optimization algorithms. This section will present a hybrid algorithm, as mentioned in introduction, the modification contains two parts. The first one is parameter control of PSO algorithm. The second is hybrid with crossover operator.

The main procedures of the proposed algorithm is given in Fig. 3. In this figure, crossover operator step and parameter updating step are highlighted in bold. It is well known that there are many crossover methods reported in literature. Commonly used operators are uniform crossover, binomial crossover, exponential crossover, arithmetic crossover. In this paper, arithmetic crossover is used as its solid theoretic basis. Note that nonuniform antenna problem in this paper is thinned as a binary optimization problem, hence arithmetic crossover is discretized to 0 if the value is less than 0.5 ; otherwise set to 1 .

The updating of parameters is based on the following thought. In the first stage, algorithm should focus on exploring search space and probe all candidate possible field. In the last stage, algorithm should focus on exploiting the fields with possible global optima found by the algorithm. Clearly, an intermediate stage should exist during the evolutionary process of algorithm, which may cost longer search time than the first and last stages. This is because the locating of global optima is crucial, and locating or trapping in local optimum is not wanted. In this paper, the parameters $w, c_{1}$, and $c_{2}$ are computed by:

$$
w(t+1)=w^{\max }-\left(w^{\max }-w^{\min }\right) \times \frac{1}{1+\exp (6-12 t)},
$$

$$
c 1(t+1)=c 1^{\max }-\left(c 1^{\max }-c 1^{\min }\right) \times \frac{1}{1+\exp (12 t-6)},
$$

$$
c 2(t+1)=c 2^{\max }-\left(c 2^{\max }-c 2^{\min }\right) \times \frac{1}{1+\exp (12 t-6)},
$$




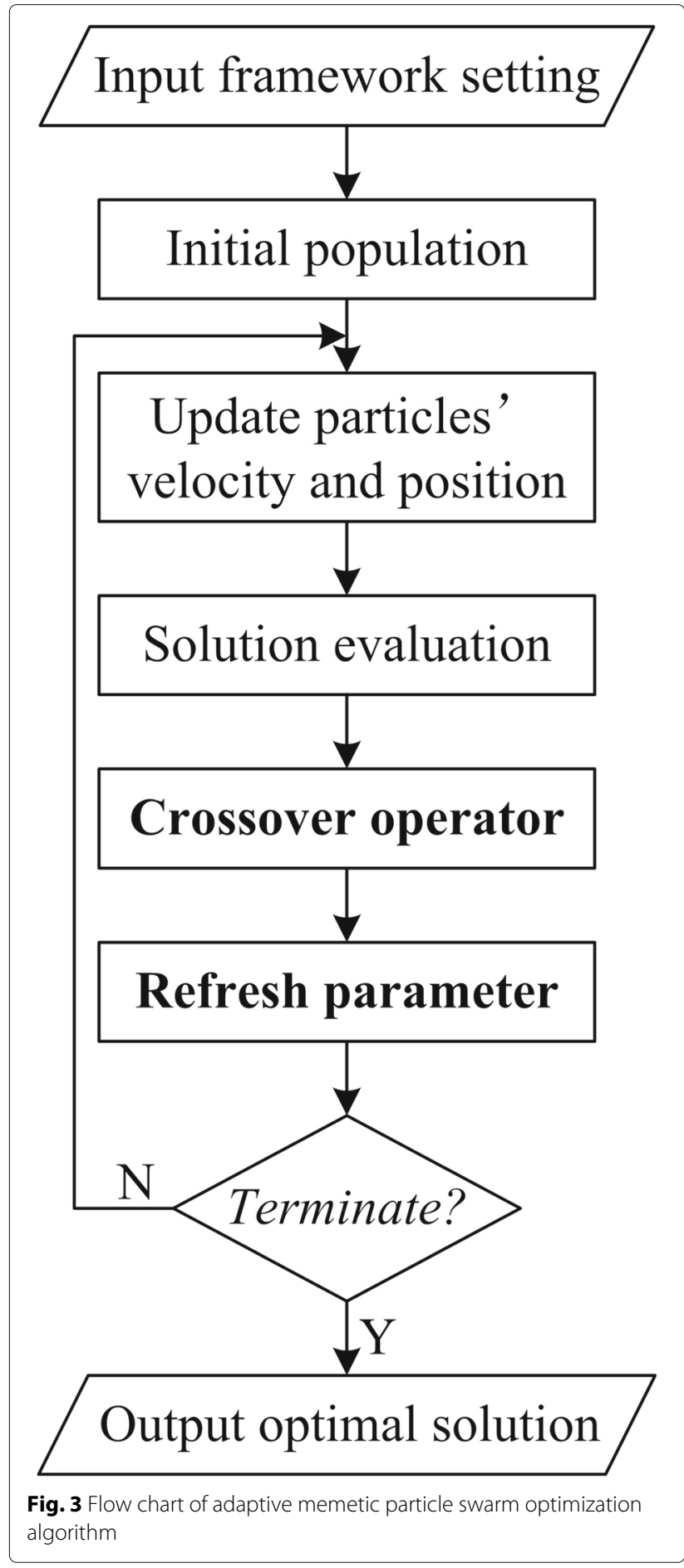

where $w^{\min }$ and $w^{\max }$ are the maximum and minimum value of parameter $w$; similarly, the minimum and maximum value of $c 1$ and $c 2$ are $c 1^{\min }, c 1^{\max }$ and $c 2^{\min }$, $c 2^{\max }$.

The adaptation of $w$ is shown in Fig. 4; the adaptation of $c 1$ and $c 2$ is given in Fig. 5. $w^{\max }$ is 0.9 and $w^{\min }$ is 0.4 . In literature, a linear decrease of $w$ from $w^{\max }$ to $w^{\min }$ is used.

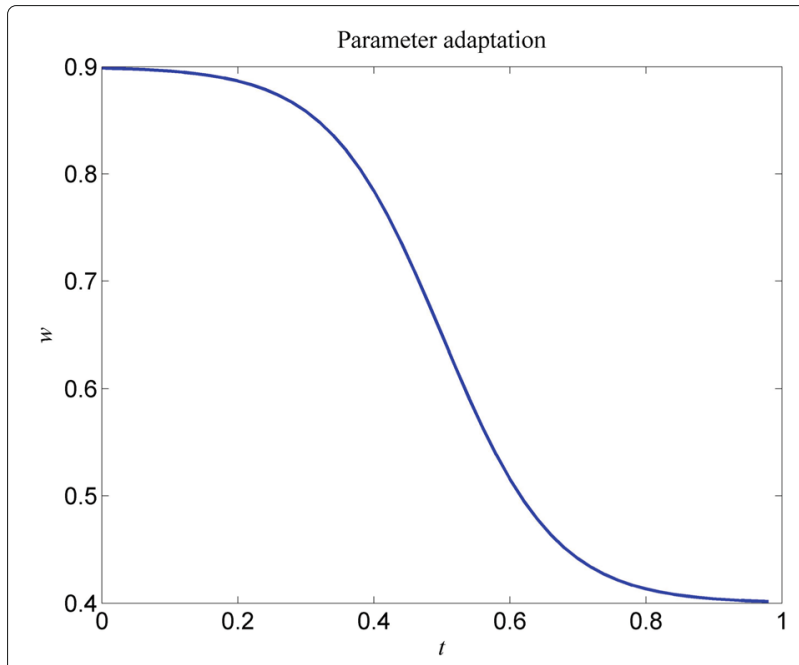

Fig. 4 Parameter adaptation for $w$

While the adaptation of $w$ in this paper is alike sigmoid function. As to $c 1$ and $c 2$, both are adapted by the same equation.

The PSO algorithm combined with crossover operator and parameter adaptation is abbreviated as AMPSO. Compared with standard PSO, the AMPSO algorithm needs more operations to perform two additional steps. For arithmetic crossover, the computing of velocity requires $2 \mathrm{D}$ addition and $2 \mathrm{D}$ multiplication operations; the computing of position also requires $2 \mathrm{D}$ addition and 2D multiplication operations. Thus, in each generation, AMPSO needs 8D computer operations than standard PSO.

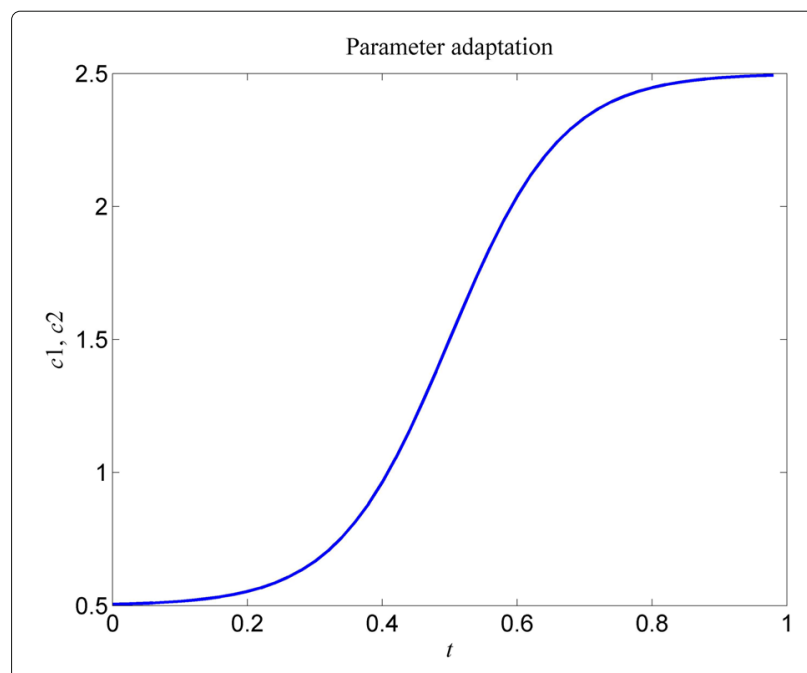

Fig. 5 Parameter adaptation for $c 1$ and $c 2$ 


\section{Numerical experiment}

The AMPSO algorithm deals with nonuniform antenna array design problem in this section. The numerical results are also reported and discussed.

\subsection{Experimental setting}

Electromagnetic phenomena of antenna array systems can be described using Maxwell's electromagnetic field equations, thus numerical methods of electromagnetic field computation, such as finite element method (FEM), method of moments, Monte Carlo method, and finite volume method, are in a better position for engineers to appreciate and analyze the performance antenna array systems. FEM is used in this paper to simulate antenna array system.

Simulation configuration is described as follows. Nonuniform antenna array design are thinned and then used to study how AMPSO performs in handling binary optimization design problems. The number of elements is $25(N=25)$, and the number of grids is $101(D=$ $M=101)$. The interval between elements is $0.5 \lambda$ and the aperture is $50 \lambda$.

Standard PSO and the proposed AMPSO are applied to tackle the above design example. In addition, since two methods are used to improve PSO, it is necessary to identify if both could shed positive effect on PSO algorithm. PSO memetic with arithmetic crossover is denoted as MPSO. The configurations of the test algorithms are shown in Table 1. The AMPSO algorithm contains less parameters than PSO. As $D$ is very large, $N p$ is set to 100 based on our empirical experience.

As to termination condition, the maximum number of function evaluations (MFE) is set to $5 \mathrm{e} 4$ for all algorithms, i.e., $M F E=50,000$. Each algorithm is independently run 25 times to gain an average performance. The simulation is implemented in Matlab, and executed on a personal computer with 4-core $3.4 \mathrm{GHz} \mathrm{CPU}$ and $4 \mathrm{~GB}$ of memory. This could provide a fair comparison environment for the test algorithms.

\subsection{Simulation results}

The optimal objective function values attained by each algorithm is shown in Table 2. Over 25 independent runs, min denotes the minimum values; similarly, med, max and mean are respectively the median, maximum and mean values; std denotes standard deviation of values. Table 2

Table 1 Configuration of the PSO, MPSO, and AMPSO algorithms

\begin{tabular}{cl}
\hline Algorithm & Parameters \\
\hline PSO & $N p=100, w=0.8, c 1=c 2=2$ \\
MPSO & $N p=100, w=0.8, c 1=c 2=2, c r=0.8$ \\
AMPSO & $N p=100, C r=0.8$ \\
\hline
\end{tabular}

Table 2 Optimal $f$ values found by the test algorithms for antenna design

\begin{tabular}{cccccc}
\hline Algorithm & $\min$ & med & $\max$ & mean & std \\
\hline PSO & -10.2747 & -9.7138 & -9.3686 & -9.7785 & 0.2624 \\
MPSO & -11.0568 & -10.5446 & -10.1880 & -10.5823 & 0.2897 \\
AMPSO & -11.3935 & -10.7617 & -10.4727 & -10.8028 & 0.2619 \\
\hline
\end{tabular}

contains the five metrics of results found by all algorithms. It is observed from Table 2 that MPSO outperforms PSO in terms of min, med, max, and mean; while the std of results of MPSO is slightly greater than that of PSO. This means that memetic with crossover may cause large variance and increase unstable factor of PSO algorithm; however, the results becomes more stable with parameter adaptive method. Compared with PSO, AMPSO attains better results in all five metrics. Moreover, AMPSO also outperforms MPSO in all five metrics. This means that both crossover operator and parameter adaptation are useful for improve the performance of PSO. Both methods can have positive effect on the algorithm.

The optimal result for thinning nonuniform antenna array by the AMPSO algorithm is shown in Fig. 6. This figure gives the far field pattern plot of Azimuth angle in degree versus PSLL in $\mathrm{dB}$. It can be seen that the difference between mainlobe and sidelobe is more than $11 \mathrm{~dB}$, which shows a high gain and satisfies the requirements of such design. In addition, sidelobe near $80^{\circ}$ and $100^{\circ}$ are depressed below to $20 \mathrm{~dB}$. This is useful for reducing signal interference between different array elements.

Figure 7 presents the convergence graphs of the best results for the PSO, MPSO, and AMPSO algorithms. It shows the best run of each algorithm over 25 repeated trials. As the curves after 10,000 function evaluations

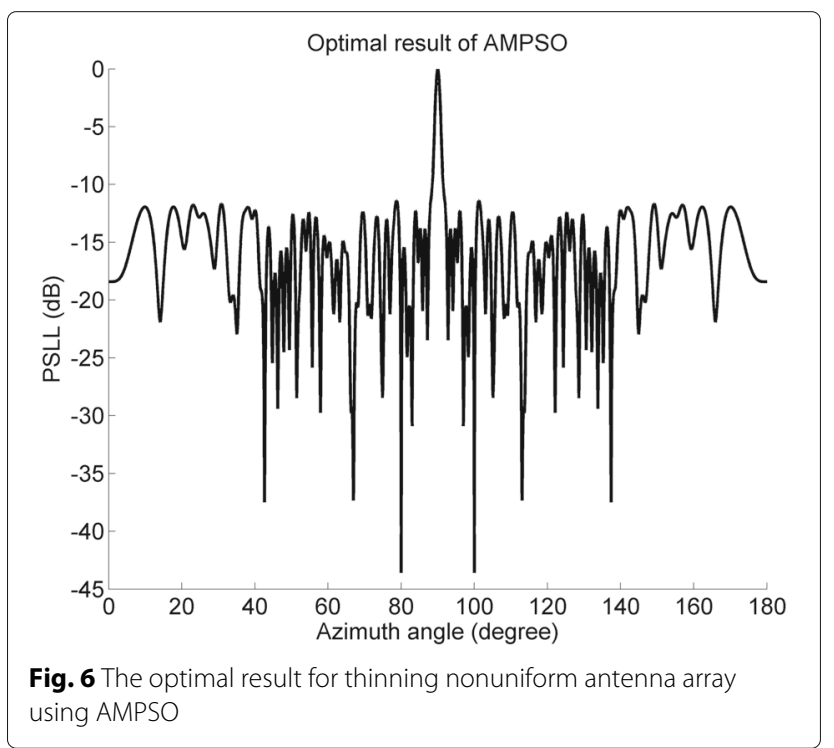




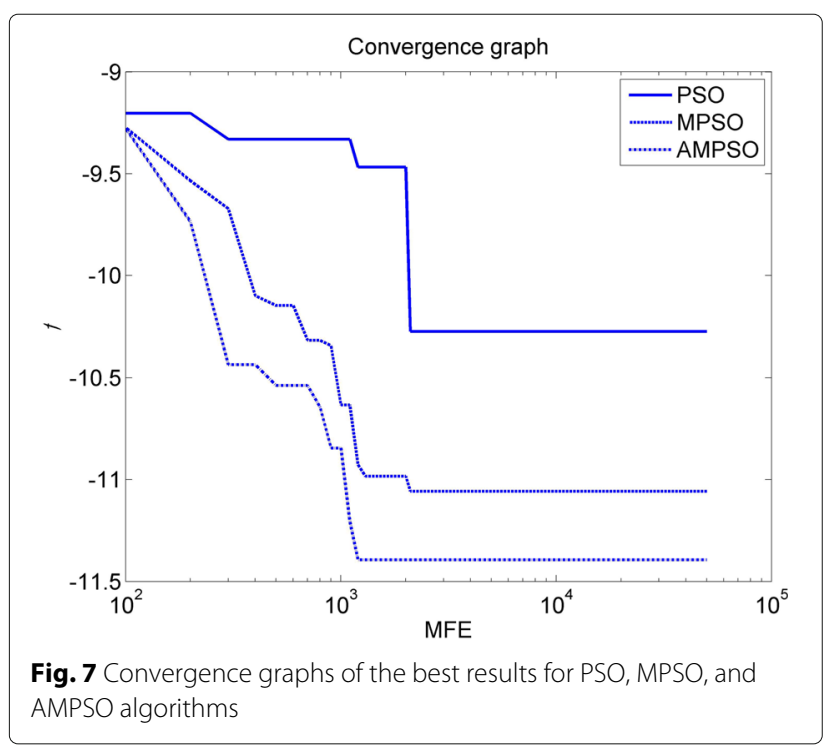

(FEs) are flat, hence, the $x$ axes are shown in log scale. For solving real world design applications, this means that less budget should be set by users. After 1000 FEs, the gap between algorithms becomes larger, which means that the proposed method improves the performance of PSO. Moreover, both the addition of crossover operator and parameter adaptation enhance the algorithm's performance. Equipped with both methods, the algorithm attains the best solution. Thus, the proposed method explores more than standard PSO and MPSO, and finally locates better solution.

\section{Conclusions}

This paper proposes to add crossover operator and parameter adaptation to standard PSO algorithm for tackling the design of nonuniform antenna array. The design of antenna array is thinned to a finite candidate slots. The increase of slot numbers brings enormous difficulties to optimization algorithms. Hence, it is necessary to study more efficient algorithms for such design problems. This paper takes arithmetic crossover and uses rounding method to discretized real values to binary values. Moreover, a novel parameter adaptation method is proposed. Different from traditional linear decrease of parameter values, parameters are adapted alike to sigmoid function. In this way, sensitive parameters are adapted and could be applied to a good many of problem types. Simulated on an example with $D=101$, the proposed AMPSO algorithm presents good performance in both convergence process and solution quality. Moreover, both the use of crossover and parameter adaptation are shown to be able to positively affect the algorithm.

In antenna array model (4), far field pattern is considered, which is the main metric in evaluating the performance of antenna. The larger the difference between main lobe beam and sidelobe beam, the higher the transmission efficiency is. However, the bandwidth of main lobe beam is not included in this model. Large bandwidth antenna is more reliable in receiving signals in complex terrain and severe weather. This issue will be studied in the future.

\section{Acknowledgements}

This research was supported in part by the National Science Foundation of China (Project Nos. 61601329, 61603275) and the Applied Basic Research Program of Tianjin (Project Nos. 15JCYBJC52300, 15JCYBJC51500).

\section{Authors' contributions}

$\mathrm{XZ}$ proposes the modified PSO method and writes most of this paper. $X Z$ is in charge of numerical simulation and proofreading of the paper. Both authors read and approved the final manuscript.

\section{Competing interests}

The authors declare that they have no competing interests.

\section{Publisher's Note}

Springer Nature remains neutral with regard to jurisdictional claims in published maps and institutional affiliations.

Received: 5 August 2017 Accepted: 20 October 2017

Published online: 06 November 2017

References

1. F Zhao, W Wang, H Chen, Q Zhang, Interference alignment and game-theoretic power allocation in MIMO heterogeneous sensor networks communications. Signal Proc. 126, 173-179 (2016)

2. J Gao, C Yin, Analysis of the local delay with slotted-aloha based cognitive radio ad hoc networks. EURASIP J. Wirel. Commun. Netw. 2015(1), 1-6 (2015)

3. Q Liang, Radar sensor wireless channel modeling in foliage environment: UWB versus narrowband. IEEE Sensors J. 11(6), 1448-1457 (2011)

4. F Zhao, $\mathrm{H}$ Nie, $\mathrm{H}$ Chen, Group buying spectrum auction algorithm for fractional frequency reuses cognitive cellular systems. Ad Hoc Netw. 58 239-246 (2017)

5. T Jiang, W Zang, C Zhao, J Shi, An energy consumption optimized clustering algorithm for radar sensor networks based on an ant colony algorithm. EURASIP J. Wirel. Commun. Netw. 2010, 627253 (2010)

6. HK Min, MS Song, I Song, JH Lim, A frequency-sharing weather radar network system using pulse compression and sidelobe suppression. EURASIP J. Wirel. Commun. Netw. 2016, 100 (2016)

7. Q Liang, Situation understanding based on heterogeneous sensor networks and human-inspired favor weak fuzzy logic system. IEEE Systems J. 5(2), 156-163 (2011)

8. F Zhao, X Sun, H Chen, R Bie, Outage performance of relay-assisted primary and secondary transmissions in cognitive relay networks. EURASIP J. Wirel. Commun. Netw. 2014, 60 (2014)

9. Q Liang, X Cheng, S Huang, D Chen, Opportunistic sensing in wireless sensor networks: theory and applications. IEEE Trans. Comput. 63(8), 2002-2010 (2014)

10. W Li, X Shi, Y Hei, An improved particle swarm optimization algorithm for pattern synthesis of phased arrays. Progress Electromagn Res. 82, 319-332 (2008)

11. C Han, T Feng, G He, T Guo, Parallel variable distribution algorithm for constrained optimization with nonmonotone technique. J. Appl. Math. 2013(1), 401-420 (2013)

12. CY Han, FY Zheng, TD Guo, GP He, Parallel algorithms for large-scale linearly constrained minimization problem. Acta Mathematicae Applicatae Sinica, English Series. 30(3), 707-720 (2014)

13. J Yu, M Li, Y Wang, G He, A decomposition method for large-scale box constrained optimization. Appl. Math. Comput. 231(12), 9-15 (2014)

14. L Jin, Y Li, C Zhao, Z Wei, B Li, J Shi, Cascading polar coding and LT coding for radar and sonar networks. EURASIP J. Wirel. Commun. Netw. 2016, 254 (2016)

15. X Zhang, X Zhang, A non-revisiting artificial bee colony algorithm for phased array synthesis. EURASIP J. Wirel. Commun. Netw. 2017(1), 7 (2017) 
16. C Ergun, $\mathrm{K}$ Hacioglu, Multiuser detection using a genetic algorithm in CDMA communications systems. IEEE Trans. Commun. 48(8), 1374-1383 (2000)

17. HG Beyer, HP Schwefel, Evolution strategies-a comprehensive introduction. Natural Comput. 1(1), 3-52 (2002)

18. R Storn, K Price, Differential evolution - a simple and efficient heuristic for global optimization over continuous spaces. J. Global. Optim. 11(4), 341-359 (1997)

19. J Kennedy, RC Eberhart, in Proc. IEEE Int. Conf. Neural Networks, vol. 4. Particle swarm optimization (IEEE, Perth, 1995), pp. 1942-1948

20. D Karaboga, B Basturk, A powerful and efficient algorithm for numerical function optimization: artificial bee colony (ABC) algorithms. J. Glob. Optim. 39(3), 459-471 (2007)

21. Z Wu, TWS Chow, Neighborhood field for cooperative optimization. Soft Comput. 17(17), 819-834 (2013)

22. F Neri, C Cotta, Memetic algorithms and memetic computing optimization: a literature review. Swarm Evol. Comput. 2, 1-14 (2012)

23. DW Boeringer, DH Werner, Particle swarm optimization versus genetic algorithms for phased array synthesis. IEEE Trans. Antennas Propag. 52(3), 771-779 (2004)

24. P Rocca, RJ Mailloux, G Toso, GA-based optimization of irregular subarray layouts for wideband phased arrays design. IEEE Trans. Antennas Propag. 14, 131-134 (2015)

25. MD Gregory, Z Bayraktar, DH Werner, Fast optimization of electromagnetic design problems using the covariance matrix adaptation evolutionary strategy. IEEE Trans. Antennas Propag. 59(4), 1275-1285 (2011)

26. X Li, WT Li, XW Shi, J Yang, JF Yu, Modified differential evolution algorithm for pattern synthesis of antenna arrays. Prog. Electromagn. Res. 137(137), 371-388 (2013)

27. S Pang, T Li, F Dai, M Yu, Particle swarm optimization algorithm for multisalesman problem with time and capacity constraints. Appl. Math. Inf. Sci. 7(6), 2439-2444 (2013)

28. P Wang, Y Li, Y Peng, SC Liew, B Vucetic, Non-uniform linear antenna array design and optimization for millimeter-wave communications. IEEE Trans. Wirel. Commun. 15(11), 7343-7356 (2016)

29. F Zhao, L Wei, $\mathrm{H}$ Chen, Optimal time allocation for wireless information and power transfer in wireless powered communication systems. IEEE Trans. Wirel. Commun. 65(3), 1830-1835 (2016)

30. F Zhao, B Li, H Chen, X LV, Joint beamforming and power allocation for cognitive MIMO systems under imperfect CSI based on game theory. Wirel. Pers. Commun. 73(3), 679-694 (2013)

31. M Taherkhani, R Safabakhsh, A novel stability-based adaptive inertia weight for particle swarm optimization. Appl. Soft Comput. 38, 281-295 (2016)

32. SU Khan, S Yang, L Wang, L Liu, A modified particle swarm optimization algorithm for global optimizations of inverse problems. IEEE Trans. Magn. 52(3), 7000804 (2016)

33. HL Jin, JW Kim, JY Song, YJ Kim, SY Jung, A novel memetic algorithm using modified particle swarm optimization and mesh adaptive direct search for PMSM design. IEEE Trans. Magn. 52(3), 7001604 (2016)

34. DH Wolpert, WG Macready, No free lunch theorems for optimization. IEEE Trans. Evol. Comput. 1(1), 67-82 (1997)

\section{Submit your manuscript to a SpringerOpen ${ }^{\circ}$ journal and benefit from:}

- Convenient online submission

- Rigorous peer review

- Open access: articles freely available online

- High visibility within the field

- Retaining the copyright to your article

Submit your next manuscript at $\gg$ springeropen.com 